\title{
AVALIAÇÃO DA RESSECÇÃO HEPÁTICA EM PACIENTES CIRRÓTICOS COM CARCINOMA HEPATOCELULAR
}

\author{
Maurício SILVA', Angelo Alves de MATTOS ${ }^{2}$,Paulo Roberto Ott FONTES ${ }^{1,3}$, \\ Fábio Luiz WAECHTER ${ }^{1,4}$ e Luiz PEREIRA-LIMA ${ }^{1,3}$
}

RESUMO - Racional - O carcinoma hepatocelular é uma complicação que acomete pacientes que apresentam cirrose hepática. A ressecção hepática, o transplante ortotópico de fígado e a ablação percutânea constituem opções terapêuticas com o intuito curativo. Objetivo - Avaliar os resultados da ressecção hepática para o tratamento do carcinoma hepatocelular, em fígados cirróticos, em um hospital geral. Métodos Foram avaliadas as características clínicas, laboratoriais, endoscópicas e histopatológicas de 22 pacientes submetidos a ressecção hepática entre os anos de 1996 e 2005, com o intuito de se avaliar a sobrevida, a identificação de fatores prognósticos e a incidência de recidiva tumoral. Para tanto, especial atenção foi dada aos níveis séricos de bilirrubinas e alfa-fetoproteína, grau de disfunção hepatocelular (avaliado pelas classificações Child-Pugh-Turcotte e "Model for End-Stage Liver Disease" - MELD), tamanho e número de tumores, invasão microvascular e presença de lesões satélites. O nível de significância utilizado foi de $95 \%$ na análise estatística. Resultados - A média de idade dos pacientes estudados foi de 62,09 anos, sendo 17 do sexo masculino. Em 10 casos a cirrose hepática esteve associada à infecção crônica pelo vírus da hepatite $\mathrm{C}$, em 4 à combinação do uso crônico do etanol e vírus da hepatite $\mathrm{C}$, em 3 ao vírus da hepatite $\mathrm{B}$, em 2 ao uso do etanol isoladamente, em 1 com uso de medicamentos e, em 2 casos, não foi identificada a causa. Dezoito pacientes apresentaram tumor único, sendo que em 11 o tumor media menos que $5 \mathrm{~cm}$. A sobrevida variou entre 10 dias e 120 meses, com média de 33,5 meses. No final do $1^{\circ}$, $3^{\circ}$ e $5^{\circ}$ anos, identificou-se sobrevida de $61,90 \%, 16,67 \%$ e $11,11 \%$, respectivamente. Houve três óbitos nos primeiros 3 meses posteriores à ressecção hepática. Treze óbitos foram identificados após os primeiros 3 meses, sendo que 12 casos foram relacionados à recidiva e progressão da neoplasia. Um paciente faleceu no período pós-operatório imediato de novo procedimento cirúrgico para ressecção de uma recidiva tumoral. No que se refere à sobrevida e à identificação de fatores prognósticos, foi identificada relação entre sobrevida e invasão microvascular. Não foi observada diferença, com significância estatística, nas curvas de sobrevida entre os níveis séricos de bilirrubinas e alfa-fetoproteína, grau de disfunção hepatocelular, tamanho e número de lesões. Entretanto, foi identificada probabilidade de recidiva tumoral maior no grupo de pacientes que apresentava invasão microvascular no estudo histopatológico. Conclusão - Apesar do pequeno número de pacientes estudados, observaram-se maus resultados com o tratamento cirúrgico do carcinoma hepatocelular. A seleção adequada dos casos pode ser fator importante para a melhoria desse resultado.

DESCRITORES - Carcinoma hepatocelular, cirurgia. Neoplasias hepáticas, cirurgia. Cirrose hepática.

\section{INTRODUÇÃO}

O carcinoma hepatocelular (CHC) é um tumor maligno primário do fígado que se caracteriza por ser, em regra, complicação da doença hepática crônica e apresentar íntima relação com fatores ambientais, o que acarreta considerável variação em sua distribuição mundial ${ }^{(6,25)}$.

Sua incidência tem aumentado nas últimas décadas em diversos países ${ }^{(6,11,40)}$. Estimativas sugerem que 564.000 novos casos surgiram durante o ano 2000, sendo que 398.364 acometeram o gênero masculino ${ }^{(45)}$. Representando $5,6 \%$ das neoplasias que ocorrem na espécie humana ${ }^{(16)}$, encontra-se entre as 10 mais freqüentes. Acomete, fundamentalmente, fígados cirróticos, com incidência anual variando entre $2 \%$ e $6 \%$, se a doença for secundária à infecção crônica pelo vírus da hepatite $\mathrm{C}^{(15)} ; 2 \%$ e $8 \%$ nos casos em que o vírus da hepatite $\mathrm{B}$ for o agente agressor ${ }^{(4)} \mathrm{e}$, em média, $5 \%$ quando a hemocromatose hereditária estiver presente ${ }^{(14)}$. Considerando-se o uso crônico de etanol, observa-se que essas são as quatro condições que conferem os maiores riscos para o surgimento do $\mathrm{CHC}^{(7)}$.

Aliada à elevada prevalência, empresta prognóstico empobrecido aos doentes. Assim, determina sobrevida em 5 anos, menor que 5\% entre os pacientes cirróticos, se não for realizada conduta terapêutica efetiva(25).

Uma vez estabelecido o diagnóstico de CHC, torna-se fundamental que seja ofertada a conduta terapêutica mais apropriada para o paciente. De modo geral, analisam-se características relacionadas ao tumor (tamanho, número, localização) e ao paciente (condição clínica global, grau

${ }^{1}$ Transplante Hepático e Cirurgia Hepatobiliopancreática, Santa Casa de Porto Alegre; Disciplinas de ${ }^{2}$ Gastroenterologia, ${ }^{3} \mathrm{Cirurgia}$ Geral e ${ }^{4}$ Técnica Operatória, Fundação Faculdade Federal de Ciências Médicas de Porto Alegre, RS.

Correspondência: Dr. Maurício Silva - Avenida Independência, 44/1104 - 90035-070 - Porto Alegre, RS. E-mail: mauriciosilva11@yahoo.com.br 
de disfunção hepática, presença de hipertensão portal). Quando se opta por uma conduta com o intuito curativo, advoga-se a utilização do transplante ortotópico de fígado (TOF), a ressecção hepática $(\mathrm{RH})$ ou a ablação percutânea ${ }^{(8)}$.

Em relação à RH, a maioria dos grupos restringe a indicação cirúrgica para tumores únicos e, no que se refere ao tamanho do tumor, não está definido um ponto de corte exato que contraindique esse tratamento ${ }^{(8)}$. Ressalte-se que essa opção não trata a doença de base nos casos em que a cirrose esteja presente.

$\mathrm{OTOF}$ apresenta resultados satisfatórios no tratamento do $\mathrm{CHC}$ em fígados cirróticos. Essa conquista foi obtida, principalmente, através do estudo realizado por MAZZAFERRO et al. ${ }^{(29)}$, que demonstrou que, se o TOF fosse realizado em pacientes com nódulo único com até $5 \mathrm{~cm}$ ou até três lesões, com a maior medindo até $3 \mathrm{~cm}$, sem evidência de invasão linfática ou vascular, os resultados seriam semelhantes àqueles encontrados em pacientes cirróticos sem essa neoplasia. Seria, portanto, o tratamento ideal a ser oferecido aos pacientes cirróticos, acometidos de $\mathrm{CHC}$. No entanto, os problemas relacionados à captação de órgãos limitam a sua indicação.

Aspecto que deve ser considerado é que, uma vez estabelecida a indicação de TOF, fica justificada a utilização de tratamentos neo-adjuvantes durante o tempo em lista. Essa conduta se apoia em estudos que demonstram a possibilidade de progressão da neoplasia durante esse período, o que pode inviabilizar que o TOF seja realizado ${ }^{(18,23,28)}$. As opções terapêuticas utilizadas com esse propósito são, fundamentalmente, a RH, a quimioembolização e a ablação percutânea ${ }^{(27,28,29)}$. Importante mencionar que, a despeito da escolha por um método específico, o tempo em lista pode ser um fator a ser considerado para a tomada de decisão. MAJNO et al. ${ }^{(28)}$ sugerem que, quando esse intervalo for menor que 6 meses, apenas a observação desses pacientes pode ser realizada.

O objetivo desse estudo foi avaliar a efetividade do tratamento cirúrgico para o $\mathrm{CHC}$, em pacientes com cirrose hepática, em um hospital geral.

\section{MÉTODOS}

O presente estudo avalia uma coorte de pacientes cirróticos submetidos ao tratamento cirúrgico para o $\mathrm{CHC}$ entre os anos de 1996 e 2005 na Irmandade Santa Casa de Misericórdia de Porto Alegre, RS. Foram incluídos todos os casos que realizaram o procedimento com um dos médicos integrantes da Equipe de Transplante Hepático e Cirurgia Hepatobiliopancreática. Os casos em que a RH foi ofertada como tratamento para pacientes em lista de espera para o TOF também foram considerados.

Realizou-se pesquisa no setor de internação hospitalar para identificação dos pacientes. Os prontuários foram revisados para verificação das variáveis em estudo contempladas no protocolo de trabalho. Quando, eventualmente, não foi possível a identificação de todas as variáveis, houve contato com o paciente ou familiar para complementação das informações exigidas. Posteriormente, localizou-se o material enviado para análise histopatológica com o objetivo de se realizar revisão histológica das lesões, por um único patologista.
A causa da cirrose foi definida como secundária ao VHB na presença do antígeno de superfície do VHB (HBsAg), ao VHC na positividade do exame qualitativo de reação de cadeia da polimerase específico para esse vírus e secundária ao uso de álcool quando a ingesta fosse maior que $40 \mathrm{~g} / \mathrm{dia}$, durante 10 anos para homens e $20 \mathrm{~g} /$ dia, no mesmo período, para mulheres.

O grau de disfunção hepatocelular foi aferido através da classificação de Child-Pugh-Turcotte (Child) ${ }^{(37)}$ e "Model for End-Stage Liver Disease" (MELD), calculado mediante fórmula matemática que contempla os valores de creatinina, bilirrubina total (BT) e relação normalizada internacional, no endereço eletrônico da "United Network for Organ Sharing and Transplantation": www.unos.org/resources/MeldPeldCalculator.

Foram avaliados parâmetros clínicos, laboratoriais, endoscópicos e histopatológicos. Os parâmetros clínicos analisados foram idade, sexo, história de ascite e encefalopatia portossistêmica. A sobrevida foi definida como o número de meses em que o paciente viveu após a realização da RH. Recidiva tumoral (RT) foi identificada através de exames de imagens (ecografia e tomografia computadorizada). Os parâmetros laboratoriais estudados foram as dosagens séricas de albumina, BT, atividade de protrombina, creatinina e alfa-fetoproteína (AFP). A dosagem dos exames precedeu, no máximo, 1 semana a data da cirurgia, com exceção da AFP que se admitiu até 30 dias. A endoscopia digestiva alta demonstrou a presença ou ausência de varizes gastroesofágicas. Os parâmetros histopatológicos estudados foram tamanho, número, grau de diferenciação celular, presença de lesões satélites e invasão vascular.

A pesquisa foi apreciada e aprovada pelo Comitê de Ética e Pesquisa da Irmandade Santa Casa de Porto Alegre.

Nas análises de sobrevida foi utilizado o método KaplanMeier com teste Log Rank para verificar possíveis diferenças entre as curvas de sobrevida entre os grupos. Os dados foram analisados utilizando-se o software SPSS 12.0 e o nível de 5\% de significância para os testes. Para variáveis quantitativas, foram calculadas as médias e os desvios-padrão, com um intervalo de confiança definido em 95\% (IC 95\%). Para testar a associação entre variáveis categóricas foi utilizado o teste exato de Fisher.

\section{RESULTADOS}

\section{Resultados gerais}

Foram analisados os dados de 22 pacientes. Destes, 17 eram homens. A idade variou entre 48 e 80 anos, com média e desviopadrão de 62,09 e 8,35 anos, respectivamente.

A causa da cirrose foi VHC em 10 casos, VHC associada a álcool em 4, VHB em 3, álcool em 2, medicamentosa em 1 (uso crônico de alfa-metildopa) e criptogênica em 2 casos.

Em relação ao grau de disfunção hepatocelular, as classificações Child A e B contemplaram 15 e 5 casos, respectivamente. Em dois casos não foi possível estabelecer o Child devido à ausência de todos os dados para o cálculo dessa classificação.

Quando se adotou a classificação MELD, foi observada a média de 9,75.

Dezenove pacientes realizaram endoscopia digestiva alta, sendo constatada a presença de varizes esofágicas em 16 casos. 
Dezoito pacientes apresentavam lesão única, sendo que o tumor media mais de $5 \mathrm{~cm}$ em sete casos. Três pacientes apresentavam duas lesões e a neoplasia era multinodular em um caso.

Identificaram-se nove casos que contemplaram critérios para serem submetidos ao TOF. Cinco pacientes realizaram a RH durante o período em lista de espera. Entre esses, três faleceram antes da realização do TOF, um paciente realizou o transplante com boa evolução pós-operatória, sem evidência de recidiva tumoral, e um está aguardando a realização do TOF. Os demais quatro casos apresentaram contra-indicações sociais para sua realização.

Em três casos o tumor foi classificado como pouco diferenciado e em cinco, como bem diferenciado. Os demais casos apresentavam grau moderado de diferenciação celular. Invasão microvascular foi identificada em 16 e a presença de lesões satélites foi descrita em 17 casos.

\section{Sobrevida após ressecção cirúrgica}

A sobrevida dos pacientes variou entre 10 dias e 120 meses, com média de 33,5 meses (IC 95\%:12,2-50,8). Para esse desfecho, foram analisados 22 pacientes. A sobrevida em 1, 3 e 5 anos foi de $61,90 \%, 16,67 \%$ e $11,11 \%$, respectivamente. Esse resultado está representado na Figura 1.

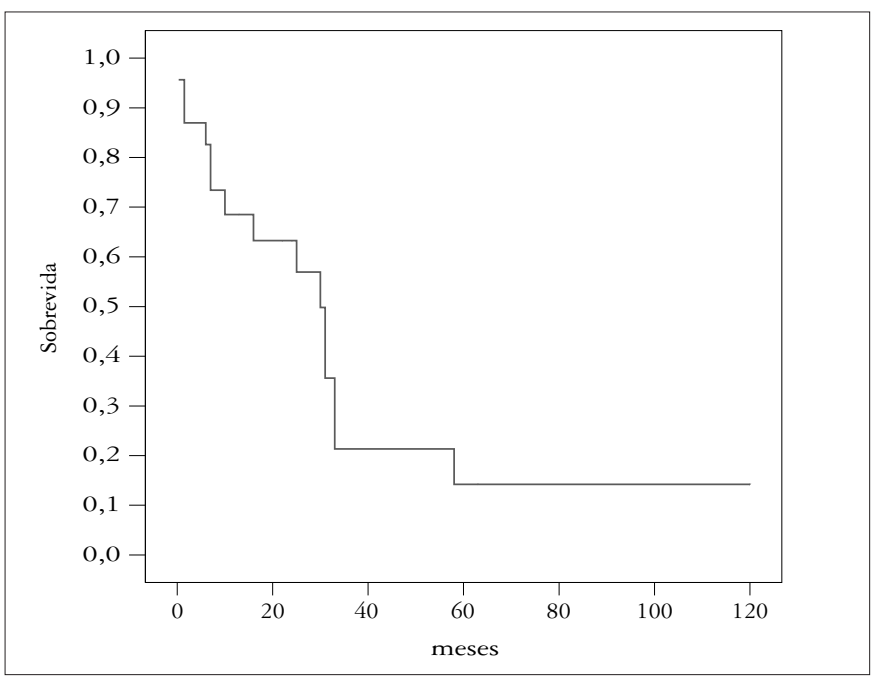

FIGURA 1. Sobrevida global após o tratamento cirúrgico

Ocorreram 16 óbitos durante o seguimento. Em três casos, esse evento aconteceu nos primeiros 3 meses (mortalidade pósoperatória imediata de 13,63\%).

\section{Identificação de fatores prognósticos}

Não se identificou diferença nas curvas de sobrevida, quando correlacionada aos níveis séricos de BT e AFP. Em relação ao grau de disfunção hepatocelular, tanto quando se estratificaram os pacientes em Child A e B, quanto os casos com MELD menor ou igual e maior que 10, também não foi identificada diferença nas curvas de sobrevida.
Quando se compararam as curvas de sobrevida dos pacientes que apresentavam lesão única menor ou igual a $5 \mathrm{~cm}$, com aqueles com lesão maior ou múltiplas, não se identificou diferença.

Por outro lado, houve diferença nas curvas de sobrevida em relação à invasão microvascular que, quando presente, determinou sobrevida de 18,6 meses (IC 95\%:10,6-26,6), contrastando com 89 meses (IC 95\%:46-132) nos casos em que esse achado não foi encontrado. Esta diferença foi relevante sob o ponto de vista estatístico $(P=0,0158)$. Já no que se refere a lesões satélites, não foi encontrada diferença $(P=0,156)$.

Esses resultados podem ser visualizados na Tabela 1 .

TABELA 1. Análise dos fatores prognósticos relacionados à sobrevida

\begin{tabular}{|c|c|c|c|}
\hline \multicolumn{2}{|l|}{ Variável } & \multirow{2}{*}{$\begin{array}{c}\text { Sobrevida em meses } \\
33,6(\text { IC95\%:13-54,2) } \\
18,2(\text { IC95\%:11,2-25,1) }\end{array}$} & \multirow{2}{*}{$\frac{P}{0,2247}$} \\
\hline $\mathrm{BT}$ & $\begin{array}{l}>1,3 \mathrm{mg} / \mathrm{dL} \\
\leq 1,3 \mathrm{mg} / \mathrm{dL}\end{array}$ & & \\
\hline AFP & $\begin{array}{l}>200 \mathrm{ng} / \mathrm{dL} \\
\leq 200 \mathrm{ng} / \mathrm{dL}\end{array}$ & $\begin{array}{c}17,8(\text { IC95\%:10-25,5) } \\
30,7(\text { IC95\%:15,9-45,4) }\end{array}$ & 0,1663 \\
\hline CHILD & $\begin{array}{l}\text { A } \\
\text { B }\end{array}$ & $\begin{array}{c}21,5(\text { IC95\%:11,5-31,5) } \\
26,3 \text { (IC95\%:7,5-45) }\end{array}$ & 0,8226 \\
\hline MELD & $\begin{array}{l}>10 \\
\leq 10\end{array}$ & $\begin{array}{c}27 \text { (IC95\%:6-48) } \\
23,4 \text { (IC95\%:12,3-34,5) }\end{array}$ & 0,9420 \\
\hline $\begin{array}{l}\text { Nódulo } \\
\text { Dois ou r }\end{array}$ & $\begin{array}{l}\leq 5 \mathrm{~cm} \\
>5 \mathrm{~cm} \\
\text { mais nódulos }\end{array}$ & $\begin{array}{c}41,4(\text { IC95\%:16,2-66,6) } \\
23,6(\text { IC95\%:6,1-41) } \\
12,6(\text { IC95\%:0,26,2) }\end{array}$ & 0,3134 \\
\hline \multicolumn{4}{|c|}{ Invasão microvascular } \\
\hline & $\begin{array}{l}\text { Presente } \\
\text { Ausente }\end{array}$ & $\begin{array}{c}18,6 \text { (IC95\%:10,6-26,2) } \\
89 \text { (IC95\%:46-132) }\end{array}$ & 0,0158 \\
\hline \multicolumn{4}{|c|}{ Lesões Satélites } \\
\hline & $\begin{array}{l}\text { Presente } \\
\text { Ausente }\end{array}$ & $\begin{array}{c}25 \text { (IC95\%:15,8-34,3) } \\
66,2 \text { (IC95\%:10,2-122,1) }\end{array}$ & 0,156 \\
\hline
\end{tabular}

\section{Recidiva tumoral}

Entre os 22 pacientes analisados, 19 foram avaliados para a RT (excluindo-se os 3 óbitos no período pós-operatório imediato). Treze casos $(68,40 \%)$ apresentaram RT, sendo que 12 faleceram devido a complicações relacionadas à progressão da neoplasia. Um paciente faleceu no $4^{\circ}$ dia após a realização de nova cirurgia para tratamento de RT.

Em 11 pacientes foi possível identificar quando ocorreu a RT, sendo que, em 9 (69,20\%), esse evento foi identificado dentro de 12 meses posteriores à RH. O tempo médio da identificação da RT foi de 8,9 meses com variação de 3 a 25 meses. Os demais seis pacientes não apresentaram RT e todos eles estavam vivos ao final do seguimento.

Quando foi avaliada a possibilidade de RT e a presença de invasão microvascular, observou-se relação com significância estatisticamente significante $(P=0,003)$. Entre os 13 pacientes que apresentaram RT, a presença de invasão microvascular foi identificada em 12 e, nos demais 6 casos sem RT, este evento foi identificado apenas em 1 caso.

Não foi identificada a associação entre a RT com a presença de lesões satélites na análise histopatológica $(P=0,071)$. Entre os 13 pacientes que apresentaram RT, a presença de lesões satélites foi identificada em 12. No entanto, este evento foi identificado em três dos demais seis casos. 


\section{DISCUSSÃO}

Diversos estudos foram realizados com o propósito de se avaliar os resultados do tratamento cirúrgico para o $\mathrm{CHC}^{(39)}$. A partir da metade da década de 90 , estudos com maior representatividade científica foram publicados, servindo, até os dias de hoje, como elementos de referência para os conhecimentos e recomendações

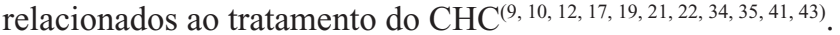

Houve inúmeros fatores que contribuíram para que os resultados da $\mathrm{RH}$ no tratamento do $\mathrm{CHC}$ apresentassem mortalidade pós-operatória e sobrevida tardia aceitáveis ${ }^{(39)}$. Entre estes, ressaltam-se o aperfeiçoamento das técnicas cirúrgicas e anestésicas $^{(42)}$, a identificação de neoplasias em fases iniciais ${ }^{(33)}$ e o aprimoramento dos conhecimentos relacionados à adequada seleção dos pacientes e dos métodos de estádio ${ }^{(8)}$.

Os resultados dos principais estudos com objetivo sobreposto ao estabelecido no presente estudo, podem ser observados na Tabela 2.

No Brasil, identificou-se um estudo onde foi avaliada a RH no tratamento do CHC publicado por HONÓRIO et al. ${ }^{(23)}$. Neste trabalho, entre os 12 pacientes analisados, observou-se média de sobrevida de 14,8 meses.

No presente estudo, a média da sobrevida encontrada foi de 33,5 meses, sendo que a mesma foi em 1, 3 e 5 anos, de $61,90 \%, 16,67 \%$ e $11,11 \%$, respectivamente. Percebe-se que a sobrevida, em todos os períodos analisados, foi menor em relação àquelas relatadas nos diferentes estudos indicados. Os resultados encontrados foram desanimadores, fundamentalmente, quando se analisou a sobrevida ao final do $3^{\circ}$ e $5^{\circ}$ anos.

Com a intenção de que fossem aprofundados os conhecimentos sobre as diferentes opções terapêuticas com intuito curativo, foi publicado, em 1999, estudo que demonstrou que o paciente ideal para ser submetido a $\mathrm{RH}$ era aquele que apresentava gradiente de pressão venosa hepática menor que $10 \mathrm{~mm} \mathrm{Hg}$, bem como valor de BT menor que $1 \mathrm{mg} / \mathrm{dL}$. Se alguma dessas variáveis estivesse presente, o paciente deveria, idealmente, ser candidato ao TOF, por apresentar maior sobrevida em 5 anos. Esse estudo considerou apenas tumores menores que $5 \mathrm{~cm}^{(26)}$.
No presente estudo, identificou-se que, entre os 11 pacientes que apresentaram nódulo único, medindo até $5 \mathrm{~cm}$, em 9 foi demonstrada a presença de hipertensão portal significativa, inferida pela identificação de varizes gastroesofágicas ao estudo endoscópico e, em 5 casos, os valores de BT estavam acima do limite superior à normalidade. Deste modo, mesmo avaliando casos que teoricamente poderiam ser beneficiados pela cirurgia, do ponto de vista das características do tumor, a intervenção teria sua indicação reavaliada, uma vez que o perfil dos pacientes não preenchia os critérios atualmente preconizados. Deve-se levar em conta, no entanto, ser este um estudo retrospectivo, que avaliou pacientes incluídos desde 1996, quando então os critérios de indicação cirúrgica não estavam estabelecidos como nos dias atuais.

Apesar de não haver um ponto de corte definido que contraindique a $\mathrm{RH}$ para casos de $\mathrm{CHC}$ em fígados cirróticos, o fato é que a maior parte dos grupos restringe sua indicação para casos com tumores medindo até $5 \mathrm{~cm}^{(8)}$. Do mesmo modo, sempre quando se encontra mais que uma lesão, o TOF é indicado inicialmente, desde que se respeitem os critérios para a sua realização ${ }^{(29)}$.

Ao ser estabelecido o período pós-operatório imediato de 3 meses, observaram-se 3 mortes entre os 22 pacientes estudados. Esse achado representa mortalidade de $13,63 \%$ nesse período. Diversos estudos têm demonstrado diminuição dessa mortalidade nos últimos anos. Enquanto que na década de 80 a mortalidade pós-operatória variava entre $10 \%$ e $20 \%$, estudos atuais demonstram que $5 \%$ dos pacientes falecem nesse período ${ }^{(3,13,36,44)}$. Difícil estabelecer comparações entre os resultados encontrados nesse estudo e aqueles citados pela literatura, porém fica sugerido que as características dos pacientes possam predispor a maior risco de morte nesse período. Na tentativa de identificar esses pacientes, importante estudo foi desenvolvido por BALZAN et al. ${ }^{(3)}$, demonstrando que, se o paciente apresentasse no $5^{\circ}$ dia após a realização da $\mathrm{RH}$, valor de BT maior que $50 \mu \mathrm{ml} / \mathrm{L}$ $(3 \mathrm{mg} / \mathrm{dL})$, associado à atividade de protrombina menor que $50 \%$, a probabilidade de óbito secundário à disfunção hepatocelular era de $59 \%$ em 2 meses, contrastando com apenas 1,2\% nos casos em que nenhum desses parâmetros fosse identificado.

TABELA 2. Resultado de diferentes estudos avaliando ressecção hepática no tratamento do carcinoma hepatocelular

\begin{tabular}{|c|c|c|c|c|c|c|}
\hline & \multirow[b]{2}{*}{ Número de casos } & \multirow[b]{2}{*}{ Cirrose (\%) } & \multirow[b]{2}{*}{$\begin{array}{c}\text { Mortalidade } \\
\text { pós-operatória (\%) }\end{array}$} & \multicolumn{3}{|c|}{ Sobrevida (\%) } \\
\hline & & & & 1 & $\begin{array}{c}3 \\
\text { anos }\end{array}$ & 5 \\
\hline Franco, $1990^{(20)}$ & 72 & 100 & 7,0 & 68 & 51 & \\
\hline Suenaga, $1992^{(41)}$ & 118 & 83 & 11,0 & 58 & 37 & 20 \\
\hline Fuster, $1996^{(21)}$ & 48 & 100 & 4,0 & 79 & 64 & \\
\hline Mazziotti, $1998^{(31)}$ & 238 & 100 & 4,6 & & & 41 \\
\hline Fong, $1999^{(19)}$ & 154 & 35 & 5,0 & 77 & 47 & 37 \\
\hline Chiappa, $2000^{(10)}$ & 51 & 100 & 8,0 & 89 & 53 & \\
\hline Grazi, 2001 $1^{(22)}$ & 264 & 100 & 4,9 & & 63 & 41 \\
\hline Poon, $2002^{(35)}$ & 206 & 100 & 6,2 & 77 & 53 & 41 \\
\hline Ercolani, $2003^{(12)}$ & 224 & 100 & 3,5 & 83 & 63 & 43 \\
\hline Vivarelli, 2004(43) & 79 & 100 & 3,8 & 83 & 65 & \\
\hline Capussotti, $2005^{(9)}$ & 216 & 100 & 8,3 & & 51 & 34 \\
\hline Ferrero, $2005^{(17)}$ & 241 & 100 & 7,9 & 76 & 52 & 37 \\
\hline
\end{tabular}


A tentativa de se obter parâmetros relacionados ao prognóstico que predisponham a melhor sobrevida em pacientes cirróticos com CHC e submetidos a RH é objetivo constante. Entre os diversos parâmetros elencados na literatura, salienta-se o papel dos níveis séricos de BT e AFP, o grau de disfunção hepatocelular, o tamanho e número de lesões e a presença de lesões satélites e de invasão microvascular ${ }^{(8)}$. Não se encontraram resultados com significância estatística nas curvas de sobrevida, no momento em que essas variáveis foram analisadas, salvo quando se avaliou a presença de invasão microvascular, observando-se pior prognóstico em relação aos casos em que esse evento ocorreu, quando comparados ao grupo sem esse achado.

A presença de invasão microvascular é importante fator que, independente da presença de cirrose hepática, empresta mau prognóstico aos doentes ${ }^{(19,21,24,46)}$. Desta forma, vai-se ao encontro da opinião de autores que sugerem ser este o principal fator prognóstico no que se refere à sobrevida ${ }^{(20,32,33,38)}$.

Não se encontrou associação entre as outras variáveis estudadas. Entre os motivos para esse achado, entende-se ser relevante o fato de que, apesar de ser número representativo de casos para apenas uma equipe cirúrgica, sob o ponto de vista epidemiológico, esse número pode ser pouco expressivo, o que certamente gera reflexos na avaliação estatística.

A RT é aspecto que deve ser avaliado quando são estudados os resultados de diferentes tratamentos relacionados a neoplasias. A despeito de a RH oferecer, em alguns casos, sobrevida em 5 anos sobreposta àquela encontrada com o TOF, diferentes estudos demonstram que a RT é bem maior, ao redor de $70 \%$, quando se considera o período de 5 anos após a cirurgia ${ }^{(1,26,34)}$.

No presente estudo, entre os 13 pacientes que apresentaram RT, observa-se que, em 9, o diagnóstico foi estabelecido no $1^{\circ}$ ano de seguimento. Estudos demonstram que, quando a RT ocorre dentro dos primeiros 3 anos, a possibilidade de disseminação local do tumor deve ser primariamente considerada ${ }^{(24,32)}$, o que, mais uma vez, pode refletir os critérios de seleção utilizados na época da cirurgia.

As reflexões que surgem sobre esse aspecto claramente reforçam o conhecimento de que o TOF é o tratamento ideal para CHC em pacientes cirróticos, pois além da remoção da neoplasia, é removido também o restante do parênquima hepático com eventuais tumores incipientes, diminuindo consideravelmente a possibilidade de RT ou surgimento de novos tumores ${ }^{(7,8)}$.

Estudos sugerem que a presença de lesões satélites e invasão microvascular são os principais fatores predisponentes ao surgimento de $\mathrm{RT}^{(1,26,32,34,38)}$. Na presente série, possivelmente em decorrência do número de pacientes estudados, apenas se constatou uma associação com a presença de invasão microvascular.

O TOF é realidade em diversos Estados brasileiros, porém, devido ao longo tempo de espera a que os pacientes estão submetidos, fica justificada a utilização da RH com o intuito de que seja evitada a progressão da neoplasia e a conseqüente exclusão da lista para o procedimento. No presente estudo, a RH foi utilizada com esse propósito em cinco casos, sendo que três faleceram em lista de espera por complicações relacionadas à RT, um foi submetido ao TOF, estando vivo e livre de neoplasia e um paciente aguarda o procedimento, sem evidência de RT.

Ressalta-se não haver embasamento científico consistente em se firmar a superioridade de um método a ser proposto, quando o paciente se encontra na lista de espera (RH, ablação percutânea e quimioembolização ${ }^{(27,28,30)}$. Em regra, poder-se-ia ofertar alternativa terapêutica aos pacientes quando o período de espera fosse superior a 6 meses e o método a ser empregado estaria na dependência da experiência local ${ }^{(28)}$.

Outro aspecto que deve ser contemplado quando se analisa o papel da RH, refere-se aos resultados conflitantes dos estudos. Assim, ADAM et al. ${ }^{(1)}$ publicaram estudo que demonstrou diminuição da sobrevida quando a RH foi utilizada como ponte para o TOF, em comparação ao TOF realizado sem ressecção prévia. Por outro lado, no mesmo ano, BELGHITI et al. ${ }^{(5)}$ relataram bons resultados nessa situação, sugerindo que o TOF poderia inclusive ser dispensado nos casos em que o paciente não apresentasse RT e, se esse evento ocorresse, o procedimento poderia ser indicado sem comprometer os resultados do procedimento.

O "Barcelona Clinic Liver Cancer" relatou estudo prospectivo ${ }^{(38)}$ no qual o TOF era indicado, após a RH, nos casos em que a possibilidade de RT fosse elevada (presença de nódulos satélites, tumores pouco diferenciados, invasão microvascular ou margens cirúrgicas comprometidas). Foram analisados 17 pacientes, sendo que 8 apresentavam tais características. Entre estes, em sete foi identificada a presença de RT no explante, contrastando com dois casos em que esse evento foi encontrado no grupo de baixo risco. Sugeriu-se, portanto, que o TOF deveria ser indicado, de imediato, após a RH nos casos em que fosse elevada a possibilidade de RT.

A despeito dos estudos aqui apresentados, reitera-se a importância do trabalho realizado por LLOVET et al. ${ }^{(26)}$ que demonstra, baseado na determinação do gradiente de pressão venosa hepática e no nível sérico de bilirrubinas, a melhor abordagem a ser ofertada a essa população de pacientes.

Considerando os dados obtidos com este trabalho, julga-se que, apesar do pequeno número de pacientes, o tratamento cirúrgico para o $\mathrm{CHC}$, em fígados cirróticos, não apresentou bons resultados no que se refere aos principais desfechos analisados: mortalidade pós-operatória imediata, sobrevida tardia e recidiva tumoral. A razão principal identificada é que a maior parte dos pacientes extrapolou os limites atualmente preconizados pela literatura médica no sentido de que sejam otimizados os resultados com essa alternativa terapêutica. Tornase fundamental, portanto, reflexões no sentido de se adotar uma seleção mais apropriada dos casos. 
Silva M, Mattos AA, Fontes PRO, Waechter FL, Pereira-Lima L. Evaluation of hepatic resection for hepatocellular carcinoma on cirrhotic livers. Arq Gastroenterol. 2008;45(2):99-105.

ABSTRACT - Background - The hepatocellular carcinoma is a disorder that affects patients suffering from cirrhosis. Liver resection, orthotopic liver transplantation and percutaneous ablation are some forms of therapy currently used to provide a cure for this disease. Aim - To assess the results achieved through liver resection for the treatment of the hepatocellular carcinoma in patients with cirrhosis being under treatment in a general hospital. Methods - Clinical observation, laboratory test results, endoscopic and histopathologic analysis were taken into consideration in the case of 22 patients who underwent liver resection between 1996 and 2005. To verify the survival rates, identify the prognostic factors and determine the risk of recurrence, special attention was given to the serologic levels of bilirubins and alpha-fetoprotein, and to the level of the hepatocellular dysfunction (classified according to the Child-Pugh-Turcotte and the Model for End-Stage Liver Disease parameters). The size and number of tumours, microvascular invasion and the presence of satellite lesions were also taken into consideration. The level of statistic significance employed was of $95 \%$. Results - In the cases studied, patients had an average age of 62.09 years, being 17 of them male. In 10 cases the liver cirrhosis was associated to the hepatitis $\mathrm{C}$ chronic infection; in 4 cases there was a combination of chronic ingestion of ethanol and the hepatitis $\mathrm{C}$ virus; in 3 cases there was an association with the hepatitis B virus chronic infection. Two cases were related to the chronic ingestion of ethanol alone and in one case the use of medications was reported. It was not possible to establish the etiology in two of the cases studied. Eighteen patients had a single tumour, 11 of them smaller than $5 \mathrm{~cm}$. The survival rate varied between 10 days and 120 months, being of 33.5 months on average. At the end of the $1 \mathrm{st}$, $3 \mathrm{rd}$ and 5 th year, the survival rates were $61.90 \%, 16.67 \%$ and $11.11 \%$, respectively. Three patients died within the first 3 months after the liver resection. Thirteen patients died after the first 3 months, 12 of the cases associated to tumour recurrence and tumour progression. There was one death in the immediate post-operative period of a second operation carried out to remove a recurrent tumour. When it comes to the survival rate and the identification of prognostic factors, a relationship between patient's survival and microvascular invasion was observed. No statically significant relationship was established between the survival rate and the serologic levels of bilirubins and alpha-fetoprotein or the level of hepatocellular dysfunction, size or number of tumours. However, a more significant incidence of tumour recurrence was observed in patients with microvascular invasion in the histopathologic study. Conclusion - In spite of the reduced number of cases studied, the surgical treatment of the hepatocellular carcinoma produced bad results. A careful selection of cases where surgery could be an option may be a decisive factor to improve such results. Acareful selection of cases might be a decisive factor in order to improve such results.

HEADING - Carcinoma, hepatocellular, surgery. Liver neoplasms, surgery. Liver cirrhosis.

\section{REFERÊNCIAS}

1. Adachi E, Maeda T, Matsumata T, Shirabe K, Kinukawa N, Sugimachi K, Tsuneiyoshi M. Risk factors of intrahepatic recurrence in human small hepatocellular carcinoma. Gastroenterology. 1995;108:768-75.

2. Adam R, Azoulay D, Castaing D, Eshkenazy R, Pascal G, Hashizume K, Samuel D, Bismuth $\mathrm{H}$. Liver resection as a bridge to transplantation for hepatocellular carcinoma on cirrhosis: a reasonable strategy? Ann Surg. 2003;238:518-9.

3. Balzan S, Belghiti J, Farges O, Ogata S, Sauvanet A, Delefosse D, Durand F. The "50-50 criteria" on postoperative day 5: an accurate predictor of liver failure and death after hepatectomy. Ann Surg. 2005;242:824-8.

4. Beasley RP, Hwang LY, Lin CC, Chien CS. Hepatocellular carcinoma and hepatitis B virus. A prospective study of 22707 men in Taiwan. Lancet. 1981;21:1129-33.

5. Belghiti J, Cortes A, Abdalla EK, Regimbeau JM, Prakash K, Durand F, Sommacale D, Dondero F, Lesurtel M, Sauvanet A, Farges O, Kianmanesh R. Resection prior to liver transplantation for hepatocellular carcinoma. Ann Surg. 2003;238:885-92.

6. Bosch FX, Ribes J, Diaz M, Cleries R. Primary liver cancer: worldwide incidence and trends. Gastroenterology. 2004;127 (Suppl 1):s5-16.

7. Bruix J, Sherman M, Llovet JM, Beaugrand M, Lencioni R, Burroughs AK, Christensen E, Pagliaro L, Colombo M, Rodes J, EASL Panel of Experts on HCC European Association for the study of the liver. Clinical management of hepatocellular carcinoma. Conclusions of the Barcelona-2000 EASL conference. J Hepatol. 2001;35:421-30.

8. Bruix J, Sherman M. Management of hepatocellular carcinoma. American Association for the Study of Liver Disease AASLD. Practice Guidelines Committee. Hepatology. 2005;42:1208-36
9. Capussotti L, Muratori A, Amisano M, Polastri H, Bouzari H, Massucco P. Liver resection for hepatocellular carcinoma on cirrhosis: analysis of mortality, morbidity and survival - a European single center experience. Eur J Surg Oncol. 2005;31:986-93.

10. Chiappa A, Zbar AP, Audisio RA, Leone BE, Biella F, Staudacher C. Factors affecting survival and long-term outcome in the cirrhotic patient undergoing hepatic resection for hepatocellular carcinoma. Eur J Surg Oncol. 2000;26:387-92.

11. El-Serag HB, Mason AC. Rising incidence of hepatocellular carcinoma in the united states. N Engl J Med. 1999;340:745-50.

12. Ercolani G, Grazi GL, Ravaioli M, Del Gaudio M, Gardini A, Cescon M, Varotti G, Cetta F, Cavallari A. Liver resection for hepatocellular carcinoma on cirrhosis: univariate and multivariate analysis of risk factors for intrahepatic recurrence. Ann Surg. 2003;237:536-43.

13. Fan ST, Lo CM, Liu CL, Lam CM, Yuen WK, Yeung C, WonG J. Hepatectomy for the hepatocellular carcinoma: toward zero hospital deaths. Ann Surg. 1999;229:322-30

14. Fargion S, Fracanzani AL, Piperno A, Braga M, D`Alba R, Ronchi G, Fiorelli G. Prognostic factors of hepatocellular carcinoma in genetic hemochromatosis. Hepatology. 1994;20:1426-31

15. Fattovich G, Pantalena M, Zagni I, Realdi G, Schalm SW, Christensen E, European Conserted Action on viral hepatitis (EUROHEP). Effect of hepatitis B and C virus infections on the natural history of compensated cirrhosis: a cohort study of 297 patients. Am J Gastroenterol. 2002;97:2886-95.

16. Ferlay J, Bray F, Pisani P, Parkin DM. GLOBOCAN 2000: Cancer incidence, mortality and prevalence worldwide, version 1.0. Lyon: IARC Press. 2001.

17. Ferrero A, Vigano L, Polastri R, Ribero D, Lo Tesoriere R, Muratore A, Capussotti L. Hepatectomy as treatment of choice for hepatocellular carcinoma in elderly patients. World J Surg. 2005;29:1101-5. 
18. Fisher RA, MalufD, Cotterell AH, Stravitz T, Wolfe L, Luketic V, Sterling R, Shiffman M, Posner M. Non-resective ablation therapy for hepatocellular carcinoma: effectiveness measured by intention-to-treat and dropout from liver transplant waiting list. Clin Transpl. 2004;18:502-12.

19. Fong, Y, Sun RL, Jarnagin W, Blumgart LH. An analysis of 412 cases of hepatocellular carcinoma at a Western center. Ann Surg. 1999;229:790-800.

20. Franco D, Capussotti L, Smadja C, Bouzari H, Meakins J, Kemeny F, Grange D, Dellepiane M. Resection of hepatocellular carcinomas. Results in 72 European patients with cirrhosis. Gastroenterology. 1990;98:733-8.

21. Fuster J, Garcia-Valdecasas JC, Grande L, Tabet J, Bruix J, Anglada T, Taura P, Lacy AM, Gonzales X, Vilana R, Bru C, Sole M, Visa J. Hepatocellular carcinoma and cirrhosis. Results of surgical treatment in a European series. Ann Surg. 1996;223:297-302

22. Grazi GL, Ercorlani G, Pierangeli F, Del Gaudio M, Cescon M, Cavallari A, Mazziotti A. Improved results of liver resection for hepatocellular carcinoma on cirrhosis give the procedure added value. Ann Surg. 2001;234:71-8.

23. Honorio R, Gil JZ, Giovanoni M, Francisco Junior J, Lustre W, Trivino T, Forones NM. Hepatocellular carcinoma: treatment of 23 cases. Arq Gastroenterol. 1996;32:68-71.

24. Imamura H, Matsuyama Y, Tanaka E, Ohkubo T, Hasegawa K, Miyagaya S, Sugawara Y, Minagawa M, Takayama T, Kawasaki S, Makuuchi M. Risk factors contributing for early and late phase of intrahepatic recurrence of hepatocellular carcinoma after hepatectomy. J Hepatol. 2003;38:200-7.

25. Kew MC. Hepatic tumors and cysts. In: Feldman M, Sleisenger MH, Schrschimidt $\mathrm{BF}$, editors. Sleisenger \& Fordtran's gastrointestinal and liver disease: pathology/ diagnosis/management. 6th ed. Philadelphia: WB Sauders; 1998. p.1364-7.

26. Llovet JM, Fuster J, Bruix J. Intention-to-treat analysis of surgical treatment for early hepatocellular carcinoma: resection versus transplantation. Hepatology. 1999;30:1434-40

27. LLovet JM, Mas X, Aponte JJ, Fuster J, Navasa M, Christensen E, Rodes J, Bruix J. Cost-effectiveness of adjuvant therapy for hepatocellular carcinoma during the waiting list for liver transplantation. Gut. 2002;50:123-8.

28. Majno P, Giostra E, Morel P, Hadengue A, Mentha G. Geneva liver cancer study group. Management of hepatocellular carcinoma in the waiting list before liver transplantation. J Hepatol. 2005;42(Suppl 1):s134-43.

29. Mazzaferro V, Regalia E, Doci R, Andreola S, Pulvirenti A, Bozzeti F, Montalto F, Ammatuna M, Morabito A, Gennari L. Liver transplantation for the treatment of small hepatocellular carcinomas in patients with cirrhosis. N Engl J Med. 1996;334:693-9.

30. Mazzaferro V, Battiston C, Perrone S, Pulvirenti A, Regalia E, Romito R, Sarli D, Schiavo M, Garbagnati F, Marchiano A, Spreafico C, Camerini T, Mariani L, Miceli R, Andreola S. Radiofrequency ablation of small hepatocellular carcinoma in cirrhotic patients awaiting liver transplantation: a prospective study. Ann Surg. 2004;240:900-9

31. Mazziotti A, Grazi GL, Cavallari A. Surgical treatment of hepatocellular carcinoma on cirrhosis: a Western experience. Hepatogastroenterology. 1998;45(Suppl 3):1281-7.
32. Minagawa M, Makuuchi M, Takayama T, Kokudo N. Selection criteria for repeat hepatectomy in patients with recurrent hepatocellular carcinoma. Ann Surg. 2003;238:703-10.

33. Nagasue N, Yukaya H, Ogawa Y, Sasaki Y, Chang YZ, Niimi K. Clinical experience with 118 hepatic resections for hepatocellular carcinoma. Surgery. 1986;99:694-701.

34. Okada S, Shimada K, Yamamoto J, Takayama T, Kosuge T, Yamasaki S, Sakamoto M, Hirohashi S. Predictive factors for postoperative recurrence of hepatocellular carcinoma. Gastroenterology. 1994;106:1618-24.

35. Poon RT, Fan ST, Lo CM, Liu CL, Lan CM, Yuen WK, Yueng C, Wong J. Extended hepatic resection for hepatocellular carcinoma in patients with cirrhosis: is it justified? Ann Surg. 2002;236:602-11.

36. Poon RT, Fan ST, Lo CM, Nq IO, Lui CL, Lam CM, Wong J. Improving survival results after resection of hepatocellular carcinoma: a prospective study of 377 patients over 10 years. Ann Surg. 2001;234:63-70.

37. Pugh RN, Murray-Lyon IM, Dawson JL, Pietroni MC, Williams R. Transection of the oesophagus for bleeding oesophageal varices. Br J Surg. 1973;60:646-9.

38. Sala M, Fuster J, Llovet JM, Navasa M, Sole M, Varela M, Pons F, Rimola A, Garcia-Valdecasas JC, Bru C, Bruix J, Barcelona Clinic Liver Cancer (BCLC) group. High pathological risk after resection of recurrence after surgical resection for hepatocellular carcinoma: an indication for salvage liver transplantation. Liver Transpl. 2004;10:1294-300.

39. Song TJ, Kit EW, Fong W. Hepatocellular carcinoma: current surgical management. Gastroenterology. 2004;127:248-60.

40. Stroffolini T, Andreoni P, Andriulli A, Ascione A, Craxi A, Chiaramonte M, Galante D, Manghisi OG, Mazzanti R, Medaglia C, Pilleri G, Rapaccini GL, Simonetti RG, Taliani G, Tosti ME, Villa E, Gasbarrini G. Caracteristics of hepatocellular carcinoma in Italy. J Hepatol. 1998;29:944-52.

41. Suenaga M, Nakao A, Harada A, Nonami T, Okada Y, Sugiura H, Uehara S, Takag H. Hepatic resection for hepatocellular carcinoma. World J Surg. 1992;16:97-104.

42. Vauthey JN, Klimstra D, Franceschi D, Tao Y, Fortner J, Blumgart L, Brennam M. Factors affecting long-term outcome after hepatic resection for hepatocellular carcinoma. Am J Surg. 1995;169:28-34.

43. Vivarelli M, Giuglielmi A, Ruzzenente A, Cucchetti A, Bellusci R, Cordiano C, Cavallari A. Surgical resection versus percutaneous radiofrequency ablation in the treatment of hepatocellular carcinoma on cirrhotic liver. Ann Surg. 2004;240:102-7.

44. Wayne JF, Lauwers GY, Ikai I, Doherty DA, Belghiti J, Yamaoka Y, Regimbeau JM, Nagomey DM, Do KA, Ellis LM, Curley SA, Pollock RE, Vauthey JM. Preoperative predictors of survival after resection of small hepatocellular carcinomas. Ann Surg. 2002;235:722-30

45. World Health Organization. Mortality database. WHO Statistical Information System Avaliable at: http://www.who.int/whosis. Accessed 2001

46. Zhou XD, Tang ZY, Yang BH, Lin ZY, Ma ZC, Ye SL, Wu ZQ, Fan J, Qin LX, Zheng BH. Experience of 1000 patients who underwent hepatectomy for small hepatocellular carcinoma. Cancer. 2001;91:1479-86.

Recebido em 18/1/2007. Aprovado em 9/11/2007. 